\title{
IOL - Ignobilia Oppida Lucanorum - Habitats anonymes des Lucaniens
}

Campagne de fouilles et de prospection (août 2015)

\section{Olivier de Cazanove}

\author{
(2) OpenEdition \\ Journals \\ Édition électronique \\ URL : http://journals.openedition.org/cefr/1620 \\ DOI : $10.4000 /$ cefr. 1620 \\ ISSN : 2282-5703 \\ Éditeur \\ École française de Rome \\ Référence électronique \\ Olivier de Cazanove, "IOL - Ignobilia Oppida Lucanorum - Habitats anonymes des Lucaniens », \\ Chronique des activités archéologiques de l'École française de Rome [En ligne], Italie du Sud, mis en ligne \\ le 28 octobre 2016, consulté le 13 novembre 2019. URL : http://journals.openedition.org/cefr/1620 ; \\ DOI : 10.4000/cefr.1620
}

Ce document a été généré automatiquement le 13 novembre 2019.

(C) École française de Rome 


\title{
IOL - Ignobilia Oppida Lucanorum - Habitats anonymes des Lucaniens
}

\author{
Campagne de fouilles et de prospection (août 2015)
}

\author{
Olivier de Cazanove
}

1 Une campagne de fouilles et de prospections - la troisième du nouveau programme « Ignobilia Oppida Lucanorum / Les sites anonymes des Lucaniens ", menée par l'École française de Rome en collaboration avec la Surintendance archéologique de Basilicate s'est déroulée en août 2015, pendant trois semaines. Trois objectifs ont été simultanément poursuivis: une fouille sur l'acropole de l'habitat fortifié de Civita de Tricarico ; une fouille sur l'autre site antique de la commune de Tricarico, à Serra del Cedro ; une prospection pédestre, également à Serra del Cedro.

\section{Civita di Tricarico}

2 L'acropole de Civita de Tricarico possède sa propre enceinte qui renferme une superficie de 2,5 ha - tandis que l'ensemble du site intra-muros, rappelons-le, s'étend sur 47 ha, ce qui en fait le plus grand des sites indigènes lucaniens connus. Toutefois, sur l'acropole même, l'unique superficie presque parfaitement plate est la terrasse sommitale (alt. 931,70 m) dont la surface est d'env. $4500 \mathrm{~m}^{2}$, comme le fait voir un Modèle Numérique de Terrain (MNT) en cours de réalisation. La terrasse était contrefortée, au moins du côté ouest, là où la pente est la plus abrupte, par un mur de soutènement. Son assise inférieure est faite de blocs de calcaire remployés. Cet aménagement est tardif, comme le montre l'emploi du mortier de chaux. Il contribuait à renforcer la position dominante du temple érigé vers la fin du II ${ }^{e}$ siècle av. n. è. ou au début du siècle suivant. 
Fig. 1 - La terrasse sommitale de l'acropole de Civita di Tricarico, avec l'édifice P, la domus et le petit temple.

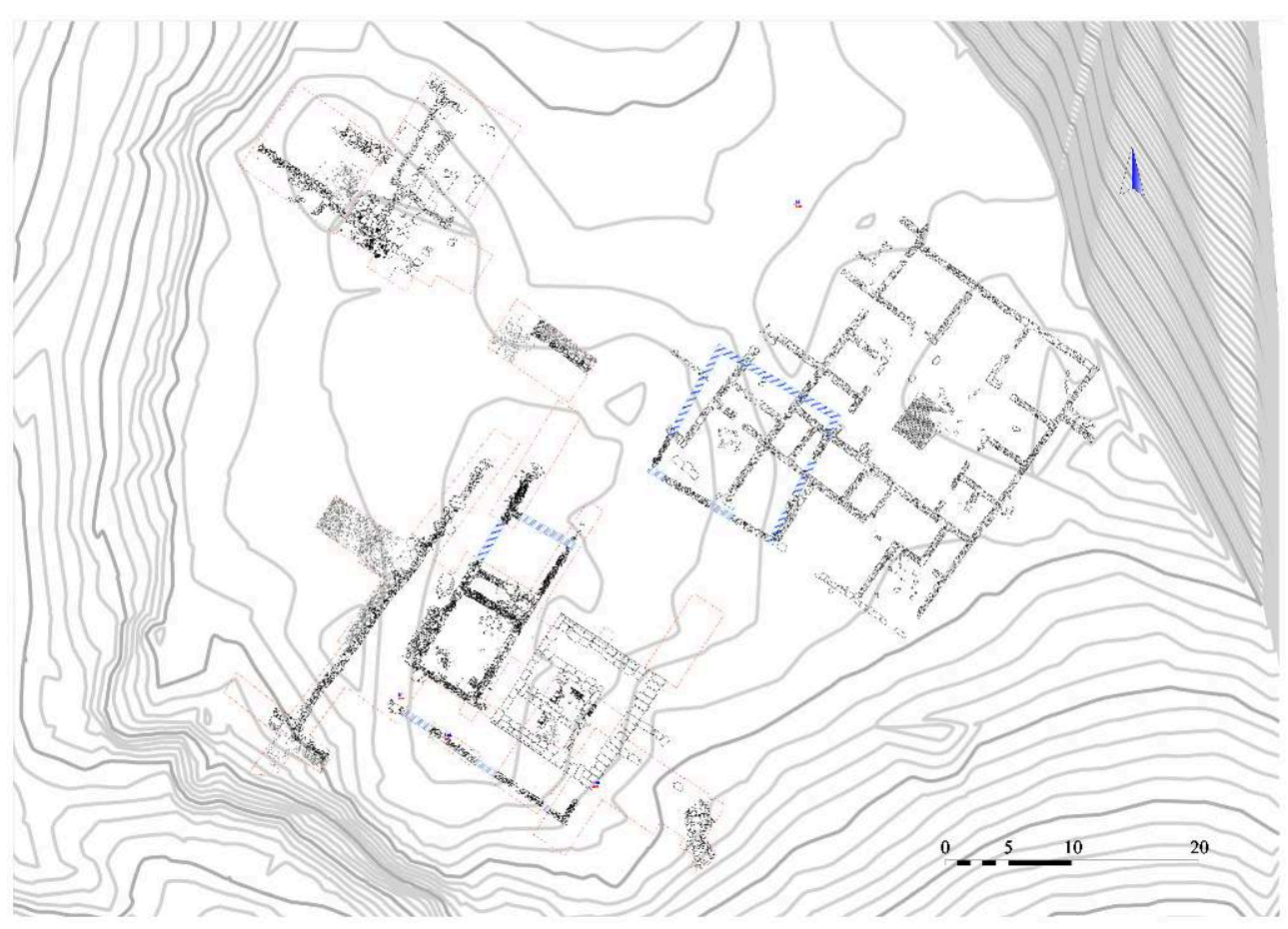

T. Terrasse et al.

3 Sur la terrasse, s'élevaient trois édifices principaux : un petit temple étrusco-italique sur podium, une domus à plan canonique, l'édifice $\pi$ qui, à leur tour, se sont superposés à des structures antérieures. Ils ont, par conséquent, des histoires constructives parallèles, qui s'éclaircissent mutuellement et qui, combinées ensemble, permettent de retracer l'évolution de ce secteur clé, en position dominante par rapport au site tout entier.

4 La domus des II $^{\mathrm{e}} \mathrm{I}^{\mathrm{er}} \mathrm{s}$. av. J.-C. a oblitéré plusieurs habitations antérieures. L'une de celles-ci, la maison $\mathrm{U}$, qui possédait une façade longue de $11 \mathrm{~m}$, a pu être restituée dans ses grandes lignes en 2014. En 2015, on a pu vérifier que la maison U avait été ellemême recouverte par une autre habitation, la maison $\mathrm{V}$, dont le côté sud atteint une longueur de $15 \mathrm{~m}$ environ. 
Fig. 2 - L'angle ouest de la maison V.

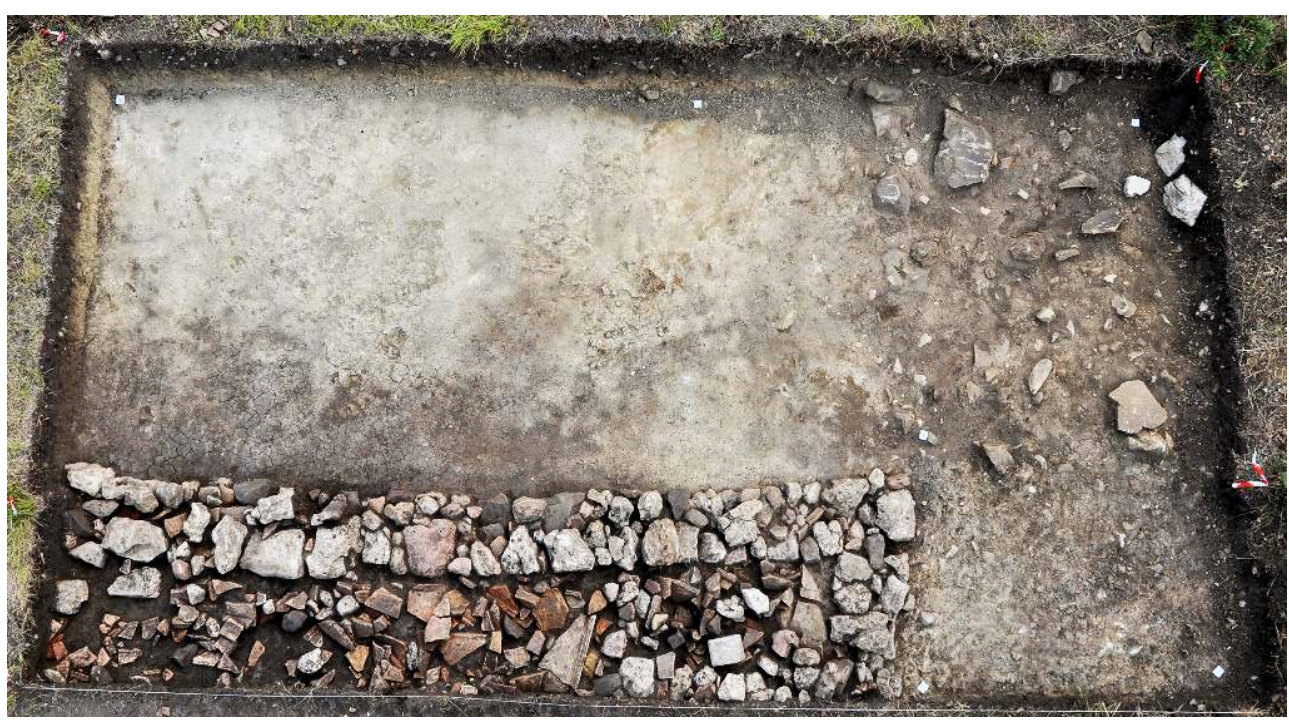

O. de Cazanove.

Cette maison, qui suit le même alignement que la domus et qui se connecte à elle au moyen d'une série de cloisons qui partent du côté nord-ouest de celle-ci, devrait toutefois être antérieure, comme l'indique la technique de construction (des murs liés à l'argile et non au mortier). L'unique pièce partiellement explorée jusqu'à présent, large d'env. 13,8 x 4.5 m (dim. internes), était peut-être la pastas. Elle était recouverte d'une épaisse strate d'écroulement de tuiles qui a dû parfaitement sceller les niveaux d'occupation. Ce «crollo» de tuiles contenait entre autres une demi-monnaie de bronze de Sagonte (inv. 5289.1: D. Rome casquée avec la légende S]AGVNT [INV. R. Proue). Il s'agit de données préliminaires, mais qui laissent entrevoir une périodisation des phases antérieures à la domus plus complexe qu'attendu.

5 L'exploration du petit temple, mis au jour en septembre 1973 par A. Fornaro pour le compte de la Surintendance archéologique de Basilicate, a repris en 2013. Il s'agissait de vérifier s'il s'était superposé à des structures antérieures. Dès la première campagne a été découvert l'édifice $\mathrm{T}$ au milieu duquel le temple a été construit. L'année suivante, en 2014, un sondage ouvert dans l'ala sud du temple a mis en lumière un mur de gros moellons informes qui passait au-dessous de la fondation de la cella. En 2015, de nouveaux sondages (au-dessous de la cella et du pronaos) ont permis, non seulement de retrouver le prolongement de ce mur, mais aussi de mettre au jour deux autres côtés de la même structure, réduits à l'état de fond de fondation en cailloutis et (partiellement) de tranchée de spoliation, bien lisible cependant. Le petit côté de la structure mesurait 3,30 m. Les côtés longs ne peuvent être restitués qu'approximativement, parce qu'ils disparaissent sous le parement sud-ouest du podium du temple - et ne réapparaissent pas de l'autre côté, alors même qu'un sondage de $4,5 \times 4 \mathrm{~m}$ avait été implanté pour en intercepter le prolongement éventuel. Par conséquent, la longueur de la petite structure peut être estimée à environ 5,5 m. 
Fig. 3 - La structure rectangulaire sous la cella e l'ala sud du temple.

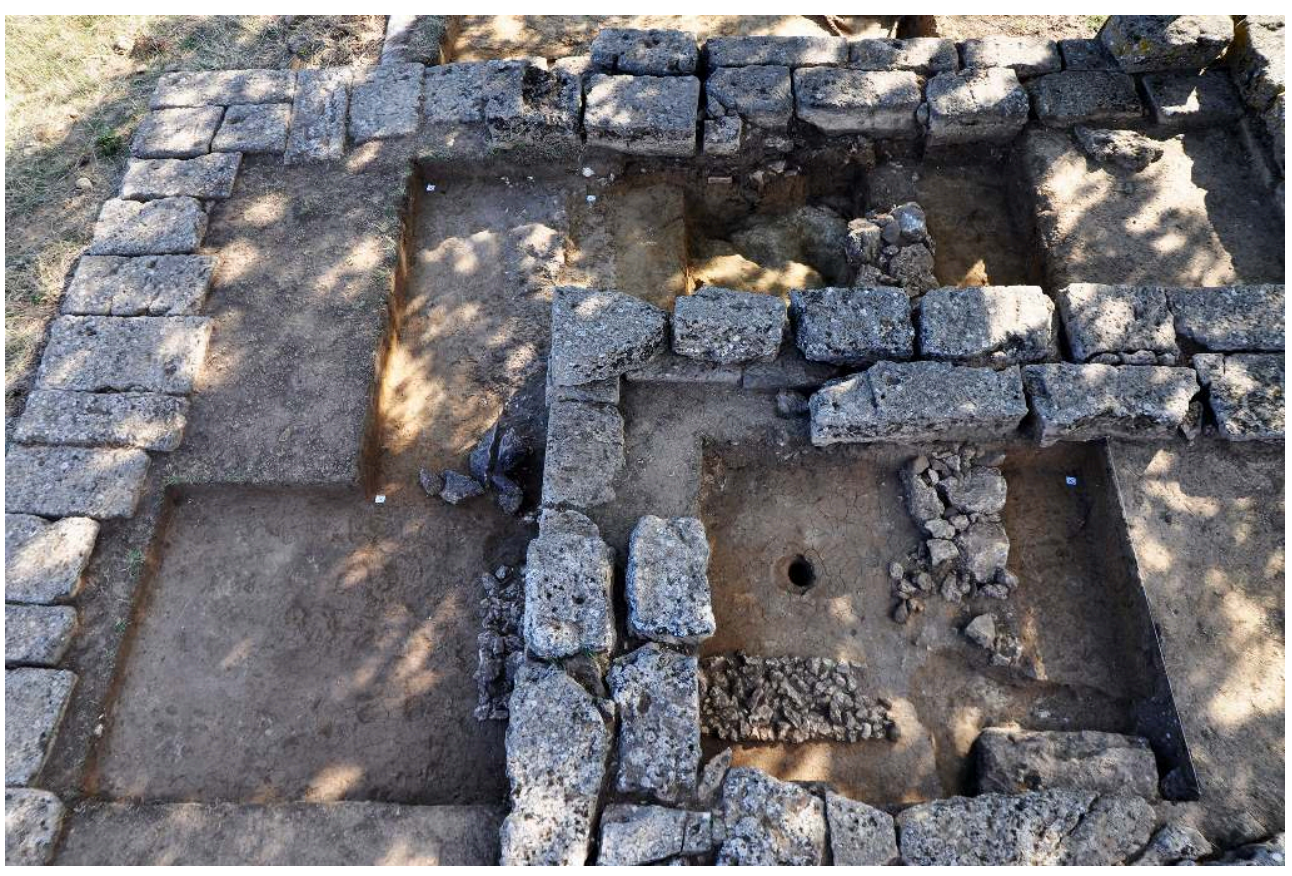

O. de Cazanove.

6 En 2016 seront effectuées d'ultimes vérifications, dans l'aile nord, la partie postérieure de la cella et derrière le temple, pour vérifier si cette structure était vraiment isolée. S'il en est ainsi, on peut avancer deux hypothèses. Il ne peut s'agir d'une habitation monocellulaire, qui serait trop petite. Cette forme rectangulaire et ces dimensions peuvent par contre faire penser à un autel. Mais il existe une autre solution : un édicule ouvert en façade sur un des côtés longs, comparable à des édifices similaires déjà fouillés à Civita de Tricarico : en particulier, le petit portique $\mathrm{N}$ et l'édifice $\mathrm{O}$, disposés de part et d'autre de la grande maison $\mathrm{M}$ au centre du plateau, tous deux publiés dans Civita de Tricarico II en 2014, et qui sont à peine plus grands. Cette solution permettrait d'expliquer la différence de technique constructive entre les différents murs de la structure retrouvée sous le temple de l'acropole: on posséderait le socle du mur du fond, en gros moellons, tandis que les hérissons de fondation correspondraient au seuil ou stylobate de l'édicule. Malheureusement, les niveaux d'occupation et le matériel associé ont en grande partie disparu. Quelques fragments pourraient toutefois renvoyer à la sphère cultuelle (fr. de fût de thymiaterion, fr. de base de louterion).

7 Une fosse a perforé les niveaux de fondation de la cella, en détruisant une grande partie de la stratigraphie antérieure. Dans le comblement ont été rejetés de nombreux fragments d'opus signinum rouge avec semis de tesselles blanches, qui devaient constituer le sol de la cella. Si le creusement de cette fosse n'est pas directement datable, on sait en revanche qu'un feu a été allumé dans le temple à la fin de l'époque byzantine ou à l'époque normande (XI ${ }^{\mathrm{e}}-\mathrm{XII}^{\mathrm{e}} \mathrm{s}$.). En effet, la datation au C14 (AMS) d'une couche noire charbonneuse dans le pronaos donne la fourchette (à deux sigmas) 1020-1160.

8 Le sondage $\pi$, fouillé par notre équipe en 1996-1998, avait révélé la superposition de deux édifices ; la façade du premier du premier d'entre eux, précédée d'un stylobate en dalles calcaires et dotée d'un large seuil avec colonne au centre, n'est pas dépourvue 
d'une certaine monumentalité. La reprise de la fouille, en 2015, visait à mettre en lumière la partie postérieure de ce complexe, non encore connue, d'en comprendre le plan et la fonction, ainsi que les liens qu'étaient susceptibles d'entretenir l'« édifice $\pi$ ", le temple et la domus.

9 Un secteur de fouille, de 9 × $8 \mathrm{~m}$, a été ouvert en 2015 immédiatement au nord-ouest du sondage $\pi$ 1996-1998. Nous avons pu avant tout établir que cette partie de la terrasse avait été utilisée dans les années 80 comme aire de travail pour la restauration de la domus fouillée en 1985-1986. Une fois enlevée cette couche contemporaine, est apparu un remblai riche de matériel du $\mathrm{II}^{\mathrm{e}} \mathrm{s}$. av. J.-C. (céramique à pâte grise en particulier). Cette couche recouvrait directement les murs. Le côté sud de l'édifice $\pi$ a été mis en lumière sur toute la longueur du sondage, mais il présente une baïonnette qui ne peut être expliquée pour l'instant. En 2016, la poursuite de la fouille permettra de préciser le plan des deux édifices superposés et leur chronologie.

\section{Serra del Cedro}

10 À Serra del Cedro, dans un vallon aux pentes raides, ont été ouvertes 2 tranchées parallèles à la ligne du gazoduc, à $4 \mathrm{~m}$ de distance de celle-ci, pour intercepter la possible prolongation des murs vus en 1986, dans une fouille préventive, lors de la mise en place de la conduite.

Fig. 4 - Serra del Cedro, mosaïque orthophotographique : sondages 2014 et 2015.

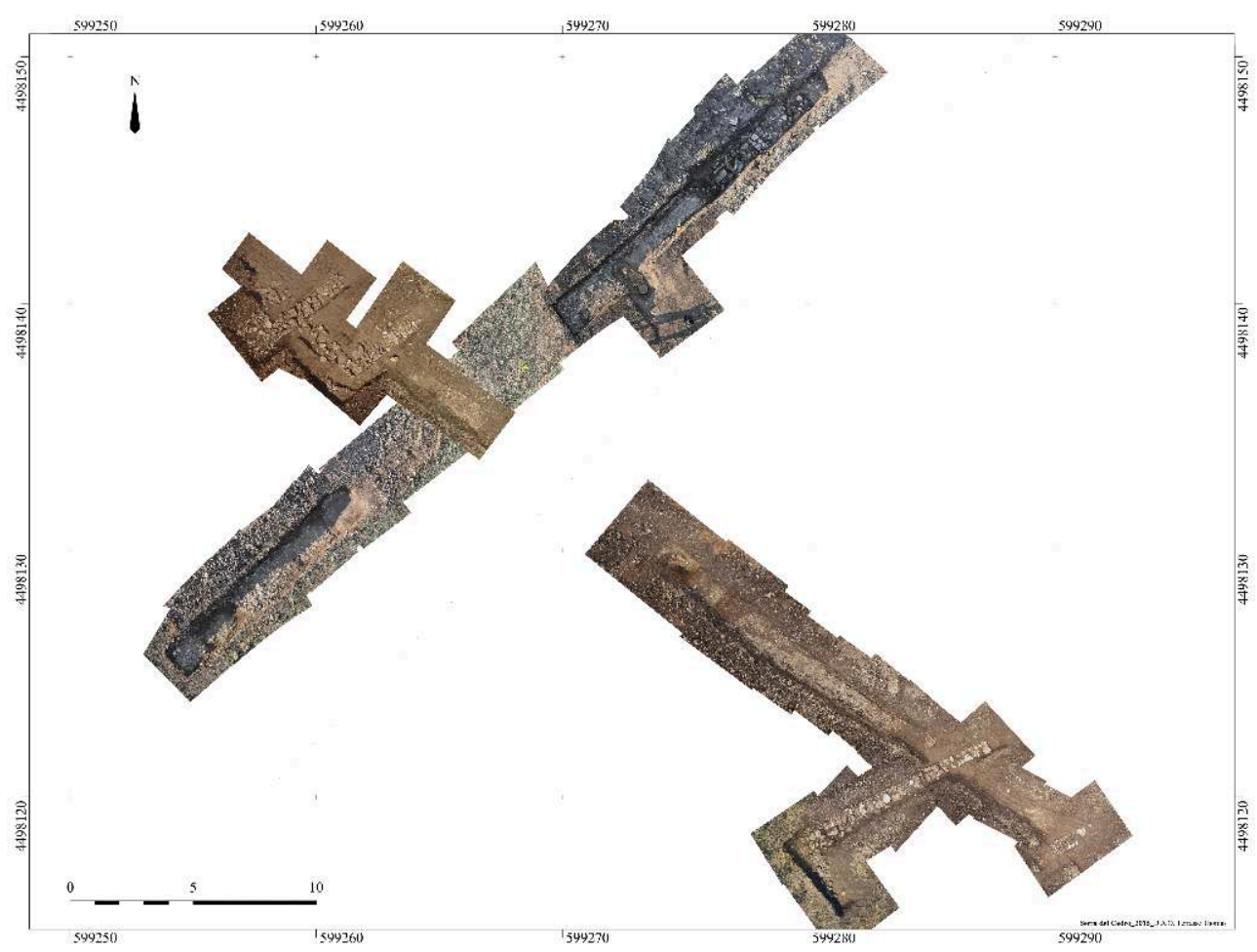

T. Terrasse

La première tranchée (a), longue de 10 x 1,5 m, n'a pas révélé de traces de structures. La deuxième tranchée (b), sur le même alignement mais $11 \mathrm{~m}$ plus en aval, a par contre 
mis en évidence une tombe et un mur de maison, qui documentent les deux phases de fréquentation de cette partie du site.

Fig. 5 - Serra del Cedro, tranchée 2.

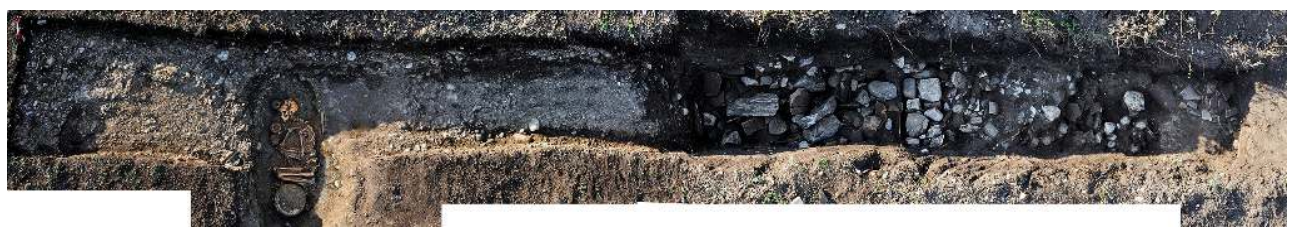

T. Terrasse.

11 La tombe SDC 1-2015, datable dans la seconde moitié de $\mathrm{VI}^{\mathrm{e}} \mathrm{s}$. av. J.-C., consiste en une fosse de 1,90 x 0,90 m, creusée dans le conglomérat à clastes arrondis («poudingue ») qui forme le sous-sol de la colline. Le squelette est celui d'un jeune adulte de sexe masculin, déposé en position fortement recroquevillée sur le côté droit, avec les bras croisés. En correspondance de la main gauche, une lame de fer oxydée semble être (dans l'attente de la restauration) celle d'un couteau. Une pointe de javelot, également en fer, était par contre disposée derrière l'individu inhumé, sur un ressaut pratiqué dans la paroi nord de la fosse. Un grand dinos à lèvre en collerette, anses en anneau plaquées contre la paroi et fond plat, reposait contre ses pieds. Quatre petits vases (des cruches) étaient déposés autour du défunt. Quatre autres vases, dont une coupe ionienne $\mathrm{B} 2$, et une lance de fer avec pointe et talon, ont été trouvées à un niveau supérieur. Elles avaient vraisemblablement été placées au-dessus du cercueil, et c'est sans doute l'affaissement de celui-ci qui a provoqué l'écrasement du crâne.

Fig. 6 - Serra del Cedro, tombe SDC 1-2015.

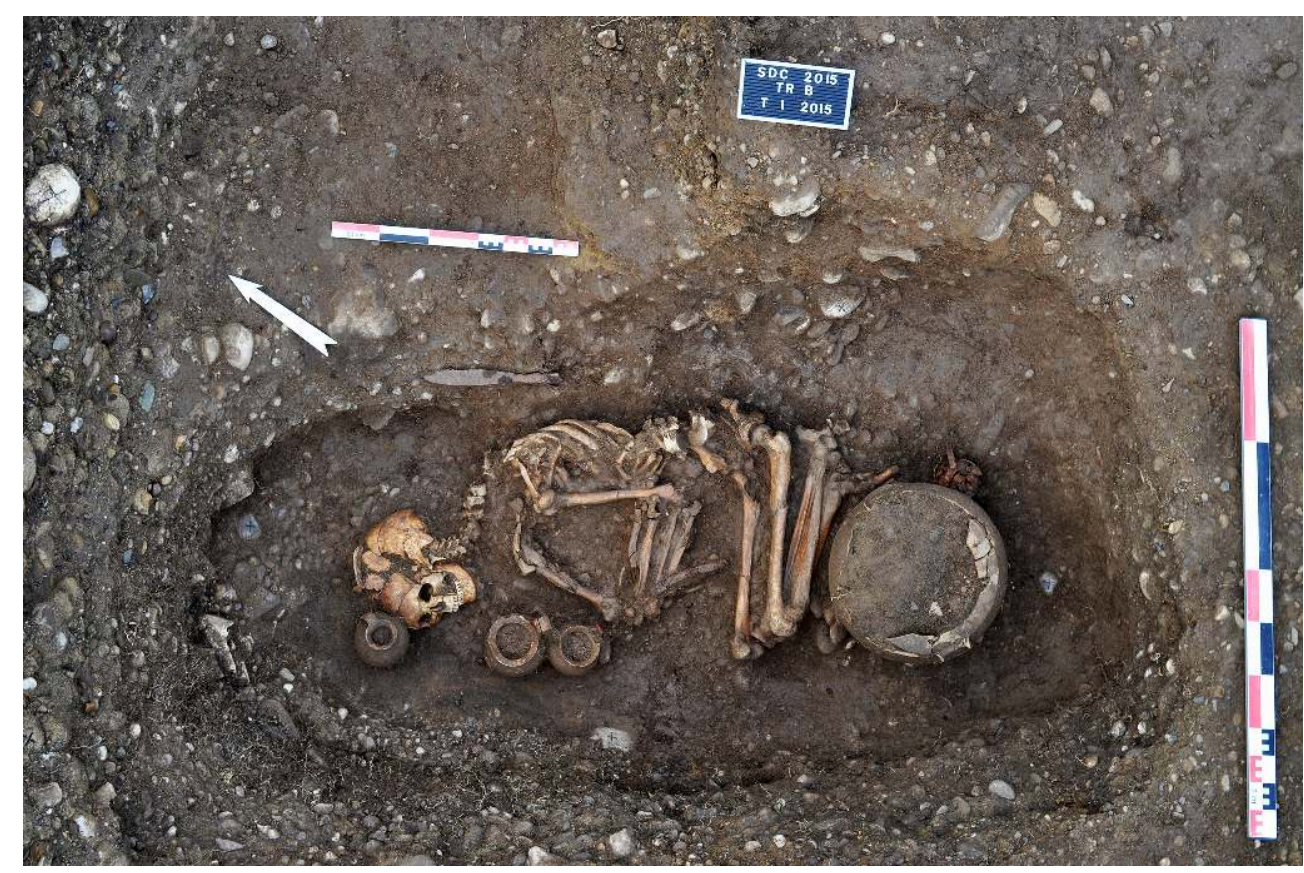

O. de Cazanove.

La tombe reste modeste, sans objets de luxe ni céramique figurée d'importation, mais elle permet de documenter en détail l'occupation de cette partie du site, à l'époque archaïque, comme nécropole (en dehors des noyaux de tombes, mieux connus, au sud 
de Serra del Cedro). Plusieurs tombes ont été fouillées par la Surintendance archéologique dans le secteur du gazoduc, et on repère malheureusement de nombreux trous de clandestins sur les deux pentes raides et dans le fond du vallon.

À $4 \mathrm{~m}$ au nord-est de la sépulture, le conglomérat naturel a été retaillé de manière à créer une terrasse en aval, pour permettre d'y édifier une maison. On a mis dans lumière en effet, à $2,6 \mathrm{~m}$ en avant de ce ressaut artificiel, un mur large de $45 \mathrm{~cm}$ (US 136), en gros galets avec des éléments de calage en grès et des fragments de tuiles creuses, conservé sur une trentaine de cm d'élévation (deux assises). De chaque côté, un dense écroulement d'écailles calcaires, de tuiles creuses, des fragments de pithoi et de vasque de louterion (US 139-140) était recouvert par une épaisse couche riche de matériel (US 129) contenant entre autres des fragments de céramique surpeinte (dite "de Gnathia »), des coupelles concavo-convexes à vernis noir, ainsi que de la céramique commune et de cuisine. La maison a donc été occupée dans la seconde moitié du IV $\mathrm{IV}_{\text {- }}$ début $\mathrm{III}^{\mathrm{e}} \mathrm{s}$. av. J.-C. Cette observation confirme les résultats de la campagne 2014 au cours de laquelle avaient été partiellement explorées trois maisons de la même période, deux d'entre elles sur le même versant et sur la terrasse immédiatement supérieure au mur mis en lumière en 2015. L'image qui commence à se dégager de ces recherches est donc celle d'une agglomération moyennement dense d'époque hellénistique, se disposant - entre autres - sur les deux versants du vallon, malgré la forte pente qui a été compensée par la création d'une série de terrasses. À cause de l'érosion, c'est toujours la partie postérieure des maisons, adossée à la pente, qui est conservée, tandis qu'a disparu la partie antérieure de celles-ci, faisant face à la pente et sans doute installée sur un terre-plein artificiel.

Des prospections pédestres ont été en outre effectuées (par L. Aniceto) sur la colline la plus à l'est du site de Serra del Cedro. En 2013, un premier survey sur le site avait révélé une importante concentration de matériel archéologique précisément dans le secteur Est, requérant une étude plus approfondie. L'objectif a été de réaliser la cartographie plus objective possible de la distribution des TCA (tuiles plates et creuses), sans extrapolations. On a choisi d'employer le système GPS de Smartphone, précis à 2-3 m près, grâce auquel chaque fragment a été géoréférencé. Ces données ont été incluses dans un SIG du site de Serra del Cedro en cours d'élaboration. Jusqu'à présent, seule la moitié est de cette hauteur (env. 1,7 ha.) a été explorée de manière systématique. On a pu ainsi comptabiliser 5377 tuiles. Un des principaux résultats de la prospection a été l'identification d'une concentration très dense dans la partie méridionale de la colline : 3357 tuiles ont été localisées sur une superficie limitée d'environ $2500 \mathrm{~m}^{2}$. Le matériel céramique, peu abondant, a été ramassé selon les différentes UT mais n'a pas été géoréférencé. Il s'agit avant tout de céramique commune ou à vernis noir, de pesons et de fragments de kalypteres hegemones, remontant au IV ${ }^{\mathrm{e}}-\mathrm{III}{ }^{\mathrm{e}} \mathrm{s}$. av. J.-C. 
INDEX

Mots-clés : Ignobilia Oppida Lucanorum, habitat, Lucanie, temple, prospection géophysique institutions École française de Rome, Université de Paris I Panthéon-Sorbonne, Labex TransferS École normale supérieure, UMR 7041 ArScAn, Soprintendenza per i beni archeologici della Basilicata

Index géographique : Civita di Tricarico, Rossano di Vaglio, Serra del Cedro

\section{AUTEUR}

OLIVIER DE CAZANOVE

Université de Paris I Panthéon-Sorbonne - cazanove[at]univ-paris1.fr 